Aims. Evidence shows that research-active trusts have better clinical patient outcomes. Psychiatric trainees are required to develop knowledge and skills in research techniques and critical appraisal to enable them to practice evidence-based medicine and be research-active clinicians. This project aimed to evaluate and improve the support for developing research competencies available to general adult psychiatry higher trainees (HT) in the North-West of England.

Method. General Adult HT in the North-West of England completed a baseline survey in November 2019 to ascertain trainee's experience of research training provision. The following interventions were implemented to address this feedback:

A trainee research handbook was produced, containing exemplar activies for developing research competencies and available training opportunities, supervisors and active research studies.

The trainee research representative circulated research and training opportunities between November 2019 - August 2020.

Research representatives held a trainee Question and Answer session in September 2020.

All General Adult HT were asked to complete an electronic survey in November 2020 to evaluate the effect of these interventions.

Result. 18 General Adult HT completed the baseline survey in November 2019. 29.4\% of trainees thought they received enough information on research competencies and $88.9 \%$ wanted more written guidance. $38.9 \%$ of trainees knew who to contact about research within their NHS Trust and $33.3 \%$ were aware of current research studies. Identified challenges for meeting research competencies included lack of time, difficulty identifying a mentor and topic and accessibility of projects.

20 General Adult HT completed the repeat survey in November 2020. 50\% of trainees wanted to be actively involved in research and 35\% wanted to develop evidence-based medicine skills. A minority of trainees aimed to complete only the minimum ARCP requirements. All trainees thought the handbook was a useful resource for meeting research competencies and would recommend it to other trainees. In trainees who received the handbook, $94.7 \%$ thought they had received adequate support on meeting research competencies and $94.7 \%$ knew who to contact about research in their trust. $68.4 \%$ of trainees would like further written guidance on meeting research competencies. Trainees highlighted ongoing practical difficulties with engaging with research and concern about lacking required skills for research.

Conclusion. Trainees are motivated to engage with research on various different levels, not purely for ARCP purposes. Simple interventions can help trainees feel adequately supported with meeting research competencies. Further work to support trainee involvement in research and improve trainee confidence in engaging with research is required.

\section{The Cambridge Mental Health Film Club: lessons to learn, feedback, expansion and development of a standard operating protocol}

Felix Clay ${ }^{*}$ Emanuele Osimo and Sara Al-Nakeeb

Cambridge and Peterborough Foundation Trust

${ }^{*}$ Corresponding author.

doi: 10.1192/bjo.2021.378

Aims. To report on our progress and feedback running the Cambridge Mental Health Film Club. To share the recent development of a Standard Operating Protocol to help others organise Mental Health Film Screenings in order to foster discussion, engage the public, reduce stigma about mental health and build understanding.

Background. Cinema lends itself to exploring social and mental health issues such as stigma in an enjoyable way within a limited time and budget. Viewing a film with those from different backgrounds and having a chance to discuss perspectives on meaning and significance is an effective way to promote a collaborative stance and expand perspectives. We have been running a Mental Health Film Club in Cambridge for the past 3 years and have recently celebrated our 10th screening.

Method. We give details of our screenings and feedback. We also share our Standard Operating Protocol which covers important topics such as resources to find suitable films, obtaining copyright permission, finding suitable venues, supporting open discussions, use of social media and promoting inclusivity.

Result. Our Mental Health Film club shows three films a year and over time has opened up to both professionals and members of the public who are interested in discussing mental health through movies and supporting recovery. We have screened many challenging and interesting films: from the impact of religious control on emergent adolescent sexuality ('The Miseducation of Cameron Post') to a classic film on Alcohol Dependency ('Days of Wine and Roses'). We also support local festivals with a similar mental health theme (e.g. MEDFEST) and have recently run a very successful screening with the University of Cambridge Psychiatry Society which was introduced by a student offering subjective experience of growing up with a sibling with an Autism Spectrum Disorder ('Life, Animated'). We promote screenings and publish all film discussions on our website (www.tinyurl.com/psychfilmclub) and Twitter in order to contribute to resources for educational use within Psychiatry training and to further involve the wider public. Feedback shows that our sessions are highly rated at helping audiences see mental health in a new way with post film discussion especially valued.

Conclusion. Our experience and practical advice can inspire others to start a Mental Health Film Club and promote cohesion, resilience and collaborative thinking within their locality. For future events we plan to expand into more public engagement via local Film Festivals. We welcome delegates ideas, experiences and film recommendations.

\section{Education for corrections officers to better meet the mental health needs of inmates}

Shaheen Darani ${ }^{1 \star}$, Kiran Patel ${ }^{1}$, Laura Hayos ${ }^{2}$, Tanya Connors ${ }^{3}$, Faisal Islam ${ }^{3}$, Anika Saiva ${ }^{4}$ and Sandy Simpson ${ }^{1}$

${ }^{1}$ University of Toronto and Centre for Addiction and Mental Health; ${ }^{2}$ Centre for Faculty Development, University of Toronto; ${ }^{3}$ Centre for Addiction and Mental Health and ${ }^{4} \mathrm{HBSc}, \mathrm{MPH}$, Centre for Addiction and Mental Health

${ }^{\star}$ Corresponding author.

doi: 10.1192/bjo.2021.379

Aims. In Canada, there has been an increase in the rate of incarceration of individuals with mental health diagnoses. Overrepresentation of individuals with psychiatric diagnoses in correctional settings is well-established. Front-line officers play a central role in dealing with mental health struggles of inmates. Nonetheless, the training that officers receive is often considered inadequate. To address this gap, the goal of this study was to design, implement, and evaluate a mental health training for 
correctional officers at the Toronto South Detention Centre (TSDC) and Vanier Centre for Women (VCW) in Ontario, Canada.

Method. A needs assessment was undertaken among officers at the TSDC. In response to needs identified, a one-day course was delivered to officers $(n=57)$ at the TSDC and VCW $(n=41)$. The curriculum included mental health awareness; assessment of risk; communicating with inmates in distress; and self-care. Live simulations provided the opportunity for participants to identify signs of mental illness, assess risk, and respond strategically to de-escalate situations. Participants' knowledge and confidence in their ability to identify and assist individuals with these problems was established using pre and post measures. Participant satisfaction was also measured via a survey. A threemonth follow-up administration was used to determine maintenance of gains. Focus groups at nine months were conducted to understand participants' needs, learning, and impact of training. Result. The results were promising, with $92 \%$ and $88 \%$ of participants at TSDC and Vanier Centre for Women respectively expressing satisfaction and $62 \%$ and $68 \%$ at TSDC and Vanier Centre for Women respectively stating they intended to change practices. Analyses of change in knowledge and confidence scores pre to post-training showed statistically significant improvement in all areas measured. Three-month follow-up at TSDC showed 75\% of respondents have applied what they learned from the training to a "moderate or great extent". Focus group themes showed improved attitudes and ability to identify behaviours related to inmate mental health struggles and interest in further training to support officers' mental health.

Conclusion. This study shows that training informed by officer learning needs can help them better meet the mental health needs of inmates. Training can improve attitudes toward inmates presenting with mental health issues. Training that is interactive and provides skills practice can have sustained impact on practice. Further training should integrate self-care to support officers' mental health.

\section{Exploring student thoughts and perception of videos as a learning resource}

\author{
Gianluca Di Pasqua* and Pranav Mahajan \\ University of Sheffield \\ ${ }^{\star}$ Corresponding author.
}

\section{doi: 10.1192/bjo.2021.381}

Aims. As medical education becomes increasingly digital, there is a plethora of readily available video resources available to medical students, aimed at teaching a wide range of topics. Despite this abundance, students report a myriad of issues. These range from videos containing outdated material, being of a poor production quality, and not being entirely relevant to their learning objectives. The aim of this study is to explore student thoughts and perceptions of videos as a teaching and learning resource. As the Mental State Examination is a component of the Psychiatry curriculum that students often find difficult, we have written, filmed and produced a video series explaining and demonstrating it.

Method. Following the production of the Mental State Examination videos at the University of Sheffield - which contained multiple doctor-patient consultations, interspersed with narration outlining the key learning points - three focus groups were undertaken. These were aimed at understanding student thoughts and perception on the new videos, and the use of videos in medical education in general. Taking a qualitative approach, thematic analysis was performed on the content of the focus groups.

Result. There was universal positive feedback about the structure and content of the videos we had produced; students enjoyed observing the various doctor-patient consultations and felt the separate elements of the Mental State Examination was explained logically and concisely. Furthermore, students appreciated that the videos were produced at their own University, believing this added to their validity. With regards to videos as a teaching resource in general, focus groups revealed that students appreciated specific, relevant and novel video material. Most students felt that videos can play an important role in medical education. There was a unanimous belief however that videos used in medical education should be produced well and be factually correct.

Conclusion. Understanding how students feel about videos as a teaching and learning resource is crucial in the development of more in the future. This study suggests more video resources for many areas, both within Psychiatry, and within the greater sphere of medical education, would be welcomed. This ought to be accompanied with student evaluation.

\section{The Safety Conversation: developing a trustwide safety conference at CNWL during a pandemic}

Emily Duncan*, Simon Edwards, Alison Butler and Cornelius Kelly

Central and Northwest London NHS Trust

${ }^{\star}$ Corresponding author.

doi: 10.1192/bjo.2021.382

Aims. The COVID pandemic has had both a massive impact on clinical service delivery and the way that training and education is provided. CNWL is a large NHS provider and has approximately 7000 staff working across 150 locations, providing mental health and community health services. In response to the need to share learning across the organisation, a trustwide "Safety Conversation Day" took place to spotlight the work being done to promote safety and to act as a platform to share ideas and learning across the trust. This was the first ever virtual conference organised by the trust.

Method. The one-day conference included virtual posters and an all-day open access virtual conversation delivered via zoom. The day was divided into 6 safety themes: Safety tools; Safer Environments; Supporting and Involving Staff; Safer use of Medicines; See Think Act and Relational Security; and Prevention is Better than Cure. Frontline staff delivered 5-6 short presentations each hour highlighting new ways of working, quality improvement, local research etc.

Staff were also encouraged to submit posters for the event, with webinars held on how to write a poster held prior to the safety conversation to promote engagement. Prizes were awarded for best posters in the different categories.

A mentimeter survey was running throughout the day to get feedback from participants.

Result. This was the largest event of this kind held by the trust. 430 unique viewers logged in during the day to watch the presentations.

Feedback was very positive on the mentimeter survey. 3 questions were asked on a likert scale of: Strongly Disagree - Strongly Agree (rated out of 5):

- 'I found the posters really useful': $4.5 / 5$

- 'I found the presentations very useful': $4.6 / 5$

- 'I will share what I've learnt about safety': 4.6/5 